\title{
Continuation of holomorphic solutions to convolution equations in complex domains
}

\author{
by RYuichi Ishimura, Jun-ICHI OKada \\ and YAsunori OKada (Chiba) \\ Dedicated to the memory of Professor Bogdan Ziemian
}

\begin{abstract}
For an analytic functional $S$ on $\mathbb{C}^{n}$, we study the homogeneous convolution equation $S * f=0$ with the holomorphic function $f$ defined on an open set in $\mathbb{C}^{n}$. We determine the directions in which every solution can be continued analytically, by using the characteristic set.
\end{abstract}

1. Introduction. In [Ki], C. O. Kiselman studied the analytic continuation of solutions of homogeneous linear partial differential equations with constant coefficients. He proved that all solutions can be continued simultaneously in the directions determined by the characteristic set of the equation. M. Zerner $[\mathrm{Z}]$ studied the domain of holomorphy of a solution to a holomorphic linear partial differential equation. Under an additional hypothesis, Sébbar [Sé] extended the results of [Ki] to the case of local differential operators of infinite order with constant coefficients. Motivated by [Sé], Aoki [A] proved a local continuation theorem for a general differential operator of infinite order with variable coefficients, using his theory of exponential calculus for pseudo-differential operators. In the preceding article [I-O1], by using the method developed in [Ki] and [Sé], we studied convolution equations with hyperfunction kernel of compact support, defined in tube domains invariant under any real translation, and we proved that the directions in which not all solutions can be continued at once are contained in the characteristic set of the operator (see also [I-O2]). For the same type of problem, we also refer for example to [V].

In this paper, we consider homogeneous convolution equations $S * f=0$ with $S$ an analytic functional on $\mathbb{C}^{n}$ and investigate the analytic continua-

2000 Mathematics Subject Classification: 32A15, 32D99, 45E10, 46F15.

Key words and phrases: analytic continuation, characteristic set, convolution equation. 
tion of the solution $f$. We first define the characteristic set $\operatorname{Char}_{\infty}(S *)$ of the equation $S * f=0$ as a natural generalization of the case of differential equations and then by the method developed in [I-O1], we evaluate the directions in which all holomorphic solutions can be simultaneously continued analytically by using the characteristic set.

2. The condition (S) and the characteristic set. For any open set $D \subset \mathbb{C}^{n}$, we denote by $\mathcal{O}(D)$ the space of holomorphic functions defined on $D$ equipped with the usual compact convergence topology. Let $S$ be an analytic functional on $\mathbb{C}^{n}$ and suppose that $S$ is carried by a compact convex set $M$. We denote by $\hat{S}(\zeta)$ the Fourier-Borel transform

$$
\hat{S}(\zeta):=\left\langle S, e^{\langle z, \zeta\rangle}\right\rangle_{z}
$$

which is an entire function of exponential type satisfying the following estimate: for every $\varepsilon>0$, there is a constant $C_{\varepsilon}>0$ such that

$$
|\hat{S}(\zeta)| \leq C_{\varepsilon} \exp \left(H_{M}(\zeta)+\varepsilon|\zeta|\right)
$$

where $H_{M}(\zeta):=\sup _{z \in M} \operatorname{Re}\langle z, \zeta\rangle$ is the supporting function of $M$. Let $\omega$ be an open set in $\mathbb{C}^{n}$. In this paper, defining the convolution by $S * f(z):=$ $\langle S, f(z-w)\rangle_{w}$, we consider the homogeneous convolution equation

$$
S * f=0
$$

with $f \in \mathcal{O}(\omega+(-M))$. We define the sphere at infinity $S_{\infty}^{2 n-1}$ to be $\left(\mathbb{C}^{n} \backslash\{0\}\right) / \mathbb{R}_{+}$and we consider the compactification by directions $\mathbb{D}^{2 n}:=$ $\mathbb{C}^{n} \sqcup S_{\infty}^{2 n-1}$ of $\mathbb{C}^{n} \simeq \mathbb{R}^{2 n}$. For $\zeta \in \mathbb{C}^{n} \backslash\{0\}$, we denote by $\zeta \infty \in S_{\infty}^{2 n-1}$ the class defined by $\zeta$, that is,

$$
\zeta \infty:=\left(\overline{\mathbb{R}_{+} \cdot \zeta} \text { in } \mathbb{D}^{2 n}\right) \cap S_{\infty}^{2 n-1} .
$$

Let $\sigma(\zeta)$ be an entire function of exponential type. (In what follows, we will take $\sigma:=\grave{\hat{S}}$, where for a function $g(\zeta)$ we set $\check{g}(\zeta):=g(-\zeta)$ ). Following Lelong and Gruman [Ll-G], we define the growth indicator $h_{\sigma}(\zeta)$ and the regularized growth indicator $h_{\sigma}^{*}(\zeta)$ of $\sigma(\zeta)$ by

$$
\begin{aligned}
& h_{\sigma}(\zeta):=\limsup _{r \rightarrow \infty} \frac{\log |\sigma(r \zeta)|}{r}, \\
& h_{\sigma}^{*}(\zeta):=\limsup _{\zeta^{\prime} \rightarrow \zeta} h_{\sigma}\left(\zeta^{\prime}\right) .
\end{aligned}
$$

As in [I-O1], and generalizing to the present case, we define the characteristic set of $S *$ : 
DeFinition 2.1. We set

$\operatorname{Char}_{\infty}(S *):=\complement\left\{\varrho \infty \in S_{\infty}^{2 n-1}\right.$ : for every $\varepsilon>0$, there exist $N>0$ and $\delta>0$ such that for any $r>N$ and $\zeta \in \mathbb{C}^{n}$ satisfying $|\zeta-\varrho /| \varrho||<\delta$, we have $\left.|\hat{S}(r \zeta)| \geq e^{\left(h_{\hat{S}}^{*}(\zeta)-\varepsilon\right) r}\right\}$,

and call it the characteristic set of the operator $S *$.

Now we recall the condition (S), originally due to T. Kawai ([Ka]) and generalized in [I-Oj].

Definition 2.2. An entire function $\sigma \in \mathcal{O}\left(\mathbb{C}^{n}\right)$ of exponential type is said to satisfy the condition $(\mathrm{S})$ in direction $\zeta_{0} \in \mathbb{C}^{n}\left(\zeta_{0} \neq 0\right)$ if it satisfies the following:

(2.5) For every $\varepsilon>0$, there exists $N>0$ such that for any $r>N$, we have $\zeta \in \mathbb{C}^{n}$ satisfying

$$
\left|\zeta-\zeta_{0}\right|<\varepsilon, \quad|\sigma(r \zeta)| \geq e^{\left(h_{\sigma}^{*}\left(\zeta_{0}\right)-\varepsilon\right) r} .
$$

REMARK 1. Ishimura and Okada [I-Oj] showed that (S) is nothing but the condition of regular growth, a classical notion in the theory of entire functions. In the case $n=1$, Ishimura [I2] constructed an example of a convolution operator which satisfies $(\mathrm{S})$ in a direction $\zeta$ but not in any (small) conic neighborhood of $\zeta$ and $\zeta \notin \operatorname{Char}_{\infty}(S *)$ (see also [I-O3]). We also remark that outside the characteristic set, the entire function $\hat{S}(\zeta)$ satisfies (S). If we make the further assumption that $h_{\hat{S}}^{*}(\zeta)$ is continuous in an open set, then the set of directions in which $\hat{S}(\zeta)$ satisfies $(\mathrm{S})$ is closed in this open set (see Theorem 4.5 of [Ll-G]). For example, assume $n=1$. In this case, $h_{\hat{S}}^{*}(\zeta)$ is always continuous (see $[\mathrm{Lv}]$ ) and so the set of directions in which (S) is satisfied is a closed set in $\mathbb{C}$. But as the above example shows, (S) is not always satisfied even if we assume the continuity of $h_{\hat{S}}^{*}(\zeta)$.

REMARK 2. By (2.1) and (2.4), we have in general $h_{\hat{S}}^{*}(\zeta) \leq H_{M}(\zeta)$. In what follows, we assume $h_{\hat{S}}^{*}(\zeta)=H_{M}(\zeta)$. For open convex domains, this last condition together with the condition $(\mathrm{S})$ is, in a sense, necessary and sufficient for the solvability of the inhomogeneous convolution equation $S * f=g$. See Krivosheev $[\mathrm{Kr}]$ for a more precise statement (see also Theorem 9.35 of $[\mathrm{Ll}-\mathrm{G}])$.

3. Division lemma. In this section, we prepare some auxiliary results. For any open set $\omega \subset \mathbb{C}^{n}$, writing $D:=\omega+(-M)$, we set

$$
\mathcal{N}(D):=\{f \in \mathcal{O}(D): S * f=0\}
$$

and equip it with the topology induced from $\mathcal{O}(D)$. 
LEMmA 3.1. Let $\sigma, \varphi$ and $\psi$ be entire functions satisfying $\sigma \varphi=\psi$, and $M$ and $K$ be two compact convex sets in $\mathbb{C}^{n}$. Suppose that for any $\varepsilon>0$, there exist constants $A_{\varepsilon}>0$ and $B_{\varepsilon}>0$ such that

$$
\begin{aligned}
\log |\sigma(\zeta)| & \leq A_{\varepsilon}+H_{M}(\zeta)+\varepsilon|\zeta|, \\
\log |\psi(\zeta)| & \leq B_{\varepsilon}+H_{K}(\zeta)+\varepsilon|\zeta| .
\end{aligned}
$$

Moreover, assume that $\sigma$ satisfies the condition (S) in every direction $\zeta_{0} \in$ $\mathbb{C}^{n} \backslash\{0\}$ and $h_{\sigma}^{*}(\zeta)=H_{M}(\zeta)$ for any $\zeta \in \mathbb{C}^{n}$. Then for any $\varepsilon>0$, there exists a constant $C_{\varepsilon}>0$ such that

$$
\log |\varphi(\zeta)| \leq C_{\varepsilon}+H_{K}(\zeta)-H_{M}(\zeta)+\varepsilon|\zeta| .
$$

REMARK 3. We will apply the lemma in the next proposition with $\sigma=\hat{S}$ and not with $\sigma=\check{\hat{S}}$.

Proof. We may prove the estimate (3.3) in each conic neighborhood of every direction $\left|\zeta_{0}\right|=1$. Set

$$
\Gamma_{\varepsilon}:=\left\{\zeta \in \mathbb{C}^{n}: \text { there exists } r>0 \text { such that }\left|\zeta-r \zeta_{0}\right|<\varepsilon r\right\} .
$$

We recall a lemma due to Harnack, Malgrange and Hörmander:

Lemma 3.2. Let $F(\zeta), H(\zeta)$ and $G(\zeta)=H(\zeta) / F(\zeta)$ be three holomorphic functions in the open ball $B(0 ; R)$. If $|F(\zeta)|<A$ and $|H(\zeta)|<B$ on $B(0 ; R)$, then

$$
|G(\zeta)| \leq B A^{2|\zeta| /(R-|\zeta|)}|F(0)|^{-(R+|\zeta|) /(R-|\zeta|)} \quad \text { for all } \zeta \in B(0 ; R) .
$$

We apply this lemma to the ball $B\left(\zeta^{\prime} ; 3 \varepsilon r\right)$. By $(3.2)$, we have

$$
\sup _{\zeta^{\prime \prime} \in B\left(\zeta^{\prime} ; 3 \varepsilon r\right)} \log \left|\psi\left(\zeta^{\prime \prime}\right)\right| \leq B_{\varepsilon}+\sup _{\left|\zeta^{\prime \prime}-\zeta^{\prime}\right| \leq 3 \varepsilon r}\left(H_{K}\left(\zeta^{\prime \prime}\right)+\varepsilon\left|\zeta^{\prime \prime}\right|\right) .
$$

Because $\left|\zeta^{\prime \prime}-r \zeta_{0}\right| \leq\left|\zeta^{\prime \prime}-\zeta^{\prime}\right|+\left|\zeta^{\prime}-r \zeta_{0}\right| \leq 4 \varepsilon r$, if we set $k:=\sup _{z \in K}|z|$, then the right hand side is estimated by

$$
\leq B_{\varepsilon}+(4 k+2) \varepsilon r+r H_{K}\left(\zeta_{0}\right) .
$$

In the same way, by setting $m:=\sup _{z \in M}|z|$ and using (3.1) we have

$$
\sup _{\zeta^{\prime \prime} \in B\left(\zeta^{\prime} ; 3 \varepsilon r\right)} \log \left|\sigma\left(\zeta^{\prime \prime}\right)\right| \leq A_{\varepsilon}+(4 m+1) \varepsilon r+r H_{M}\left(\zeta_{0}\right) .
$$

Noting that $\zeta \in B\left(\zeta^{\prime} ; 2 \varepsilon r\right) \subset B\left(\zeta^{\prime} ; 3 \varepsilon r\right)$, by Lemma 3.2 , we have

$$
\begin{aligned}
\log |\varphi(\zeta)| \leq & B_{\varepsilon}+(4 k+1) \varepsilon r+r H_{K}\left(\zeta_{0}\right) \\
& +\frac{4 \varepsilon r}{3 \varepsilon r-2 \varepsilon r}\left(A_{\varepsilon}+(4 m+1) \varepsilon r+r H_{M}\left(\zeta_{0}\right)\right) \\
& +\frac{3 \varepsilon r+2 \varepsilon r}{3 \varepsilon r-2 \varepsilon r}\left(\varepsilon-h_{\sigma}^{*}\left(\zeta_{0}\right)\right) r
\end{aligned}
$$




$$
\begin{aligned}
= & B_{\varepsilon}+4 A_{\varepsilon}+r\left(H_{K}\left(\zeta_{0}\right)+4 H_{M}\left(\zeta_{0}\right)\right) \\
& +\left((16 m+4 k+10) \varepsilon-5 h_{\sigma}^{*}\left(\zeta_{0}\right)\right) r \\
= & B_{\varepsilon}+4 A_{\varepsilon}+r\left(H_{K}\left(\zeta_{0}\right)-H_{M}\left(\zeta_{0}\right)\right) \\
& +(16 m+4 k+10) \varepsilon r
\end{aligned}
$$

for sufficiently large $r$. Therefore for any $\varepsilon>0$, we can find constants $C_{\varepsilon}^{\prime}>0$ and $N>0$ such that

$$
\log |\varphi(\zeta)| \leq C_{\varepsilon}^{\prime}+H_{K}(\zeta)-H_{M}(\zeta)+\varepsilon|\zeta|
$$

for $\zeta \in \Gamma_{\varepsilon} \cup\{|\zeta|>N\}$.

Proposition 3.3. Let $\omega$ and $\Omega$ be two open sets in $\mathbb{C}^{n}$ with $\omega \subset \Omega$. Assume that $S$ satisfies the condition (S) in every direction in $\mathbb{C}^{n}$ and $h_{\hat{S}}^{*}(\zeta) \equiv H_{M}(\zeta)$. Then the restriction map $\mathcal{N}(\Omega+(-M)) \rightarrow \mathcal{N}(\omega+(-M))$ has the dense image.

Proof. Let $E$ be the set of exponential-polynomial solutions of the equation $S * f=0$. We denote by $E^{\circ}$ the polar set of $E$ in the topological dual space $\mathcal{O}\left(\mathbb{C}^{n}\right)^{\prime}$ of $\mathcal{O}\left(\mathbb{C}^{n}\right)$. It is sufficient to prove that $E$ is dense in $\mathcal{N}(\omega+(-M))$, and for this, we will prove the following: any $T \in E^{\circ} \cap \mathcal{O}(\omega+$ $(-M))^{\prime}$ is contained in the orthogonal set $\mathcal{N}(\omega+(-M))^{\circ}$ in $\mathcal{O}(\omega+(-M))^{\prime}$. By a lemma of Malgrange (see e.g. [I-O1]), there is an entire function of exponential type $r(\zeta)$ such that $\hat{T}(\zeta)=r(\zeta) \hat{S}(-\zeta)$. By the preceding lemma, we may find an analytic functional $R$ carried by $\omega$ so that $\hat{R}(\zeta)=r(\zeta)$. Then for any $g \in \mathcal{N}(\omega+(-M))$, we have

$$
\langle T, g\rangle=\langle\check{\hat{S}} * R, g\rangle=\langle R, S * g\rangle=0,
$$

where $\check{S}$ is defined as the inverse Fourier-Borel image of $\check{\hat{S}}$.

4. Continuation of solutions of homogeneous equations. For $\operatorname{Char}_{\infty}(S *)$ and an open convex set $\omega \subset \mathbb{C}^{n}$, we now set

$$
\Omega:=\text { the interior of } \bigcap_{\zeta \infty \in \operatorname{Char}_{\infty}(S *)^{a}}\left\{z \in \mathbb{C}^{n}: \operatorname{Re}\langle z, \zeta\rangle \leq H_{\omega}(\zeta)\right\},
$$

where ${ }^{a}$ means the antipodal: $A^{a}:=-A$. By definition of $\Omega$, we know that for any compact convex set $L \subset \Omega$, there exists a compact convex set $K \subset \omega$ such that

$$
H_{L}(\zeta) \leq H_{K}(\zeta) \quad\left(\text { for any } \zeta \infty \in \operatorname{Char}_{\infty}(S *)^{a}\right)
$$

and so

$$
H_{L+(-M)}(\zeta) \leq H_{K+(-M)}(\zeta) \quad\left(\text { for any } \zeta \infty \in \operatorname{Char}_{\infty}(S *)^{a}\right) .
$$

Lemma 4.1. Assume $h_{\hat{S}}^{*}(\zeta) \equiv H_{M}(\zeta)$. Let $K$ and $L$ be two compact subsets of $\mathbb{C}^{n}$ satisfying $(4.2)$ and $p(\zeta)$ an entire function satisfying the 
estimate

$$
\log |p(\zeta)| \leq H_{L}(\zeta)
$$

Then for any $\varepsilon>0$, there exist a constant $C_{\varepsilon}>0$ and entire functions $q(\zeta)$ and $r(\zeta)$ which satisfy

$$
\begin{aligned}
& p(\zeta)=\check{\hat{S}}(\zeta) q(\zeta)+r(\zeta), \\
& \log |q(\zeta)| \leq H_{L \cup K}(\zeta)-H_{-M}(\zeta)+\varepsilon|\zeta|+C_{\varepsilon}, \\
& \log |r(\zeta)| \leq H_{K}(\zeta)+\varepsilon|\zeta|+C_{\varepsilon}
\end{aligned}
$$

for any $\zeta \in \mathbb{C}^{n}$.

Proof. We may assume $0<\varepsilon<1 / 2$. We set $\sigma(\zeta):=\check{\hat{S}}$. For any $\delta>0$, we put

$$
\begin{aligned}
& \Xi_{1}:=\left\{\zeta \infty \in S^{2 n-1}: H_{L}(\zeta)<H_{K}(\zeta)+\delta|\zeta|\right\} \\
& \Xi_{2}:=\left\{\zeta \infty \in S^{2 n-1}: H_{L}(\zeta)>H_{K}(\zeta)\right\}
\end{aligned}
$$

Then $\Xi_{1}$ is a neighborhood of $\operatorname{Char}_{\infty}(S *)^{a}$ in $S_{\infty}^{2 n-1}$ and $\Xi_{2}$ does not meet $\operatorname{Char}_{\infty}(S *)^{a}$. For large $N>0$, we set

$$
\begin{aligned}
& Z_{1}=\left\{\zeta \in \mathbb{C}^{n}: \zeta \infty \in \Xi_{1},|\zeta|>N\right\}, \\
& Z_{2}=\left\{\zeta \in \mathbb{C}^{n}: \zeta \infty \in \Xi_{2},|\zeta|>N\right\}, \\
& Z_{3}=\left\{\zeta \in \mathbb{C}^{n}:|\zeta| \leq N\right\} .
\end{aligned}
$$

For $\alpha>0$ small enough, take $\psi_{\alpha} \in C_{0}^{\infty}\left(\mathbb{C}^{n}\right)$ such that $\int \psi_{\alpha} d \zeta d \bar{\zeta}=1$, $\operatorname{supp} \psi_{\alpha}=B(0 ; \alpha)$ and $\psi_{\alpha} \geq 0$. Recalling the assumption $h_{\sigma}^{*} \equiv H_{-M}$, we set $h_{\sigma, \alpha}^{*}(\zeta):=\psi_{\alpha} * h_{\sigma}^{*}(\zeta)$, a $C^{\infty}$ function on $\mathbb{C}^{n}$. Then since $H_{-M}$ is subadditive, we have $\left|h_{\sigma, \alpha}^{*}(\zeta)-h_{\sigma}^{*}(\zeta)\right| \leq m \alpha$ with $m:=\sup _{\zeta \in M}|\zeta|$. Now we construct $q(\zeta)$ and $r(\zeta)$ as follows:

$$
\begin{aligned}
& q(\zeta):=\frac{p(\zeta)}{\sigma(\zeta)}\left(1-\varphi\left(e^{\lambda(|\zeta|)-h_{\sigma, \alpha}^{*}(\zeta)} \sigma(\zeta)\right)\right)+v(\zeta), \\
& r(\zeta):=p(\zeta) \varphi\left(e^{\lambda(|\zeta|)-h_{\sigma, \alpha}^{*}(\zeta)} \sigma(\zeta)\right)-\sigma(\zeta) v(\zeta),
\end{aligned}
$$

by choosing a suitable $C^{\infty}$ function $v(\zeta)$ on $\mathbb{C}^{n}$ (to be chosen later), with fixed real-valued functions $\varphi(\tau) \in C^{\infty}(\mathbb{C})$ and $\lambda(t) \in C^{\infty}([0, \infty[)$ so that $0 \leq \varphi(\tau) \leq 1,0 \leq \lambda(t) \leq \delta t, 0 \leq \lambda^{\prime}(t) \leq 1$ for small $\delta>0$ and that

$$
\varphi(\tau)=\left\{\begin{array}{ll}
1 & (|\tau| \leq 1 / 2), \\
0 & (|\tau| \geq 1),
\end{array} \quad \lambda(t)= \begin{cases}0 & (t \leq 1 / 2) \\
\delta t & (t \geq 1)\end{cases}\right.
$$

We first note that $q(\zeta)$ and $r(\zeta)$ are well defined: in fact, if $e^{\delta|\zeta|-h_{\sigma}^{*}(\zeta)}|\sigma(\zeta)|>$ $1 / 2-\varepsilon$, then $q(\zeta)$ is well defined since $\sigma(\zeta) \neq 0$, and if $e^{\delta|\zeta|-h_{\sigma}^{*}(\zeta)}|\sigma(\zeta)| \leq$ $1 / 2-\varepsilon$, then $q(\zeta)$ is well defined because $\varphi\left(e^{\lambda(|\zeta|)-h_{\sigma, \alpha}^{*}(\zeta)} \sigma(\zeta)\right)$ is equal to 1 . 
The condition for $q$ and $r$ to be holomorphic is the equality

$$
\begin{aligned}
\bar{\partial} v= & \frac{p(\zeta)}{\sigma(\zeta)} e^{\lambda(|\zeta|)-h_{\sigma, \alpha}^{*}(\zeta)} \\
& \times\left[\left\{\frac{\partial \varphi}{\partial \tau} \sigma(\zeta)+\frac{\partial \varphi}{\partial \bar{\tau}} \bar{\sigma}(\zeta)\right\}\left\{\frac{\lambda^{\prime}(|\zeta|)}{2|\zeta|} \zeta \cdot d \bar{\zeta}-\bar{\partial} h_{\sigma, \alpha}^{*}(\zeta)\right\}+\frac{\partial \varphi}{\partial \bar{\tau}} \bar{\partial} \bar{\sigma}\right] .
\end{aligned}
$$

We denote the right hand side by $w$; it satisfies $\bar{\partial} w=0$. We now estimate $w$. In $\complement\left\{1 / 2<e^{\lambda(|\zeta|)-h_{\sigma, \alpha}^{*}(\zeta)}|\sigma(\zeta)|<1\right\}$, we have $w=0$ as $\varphi\left(e^{\lambda(|\zeta|)-h_{\sigma, \alpha}^{*}(\zeta)} \sigma(\zeta)\right)$ is constant there. In particular, taking $N>0$ large enough, in $Z_{2}$, we have $|\zeta|>N,|\sigma(\zeta)| e^{\delta|\zeta|-h_{\sigma}^{*}(\zeta)}>1$ and so $\lambda(|\zeta|)=\delta|\zeta|, \varphi\left(e^{\delta|\zeta|-h_{\sigma, \alpha}^{*}(\zeta)} \sigma(\zeta)\right)=0$.

In $\left\{1 / 2<e^{\lambda(|\zeta|)-h_{\sigma, \alpha}^{*}(\zeta)}|\sigma(\zeta)|<1\right\}$, recalling $h_{\sigma}^{*} \equiv H_{-M}$, we first remark that for any $j$,

$$
\begin{aligned}
\left|\frac{\partial}{\partial \bar{\zeta}_{j}} h_{\sigma, \alpha}^{*}(\zeta)\right| & \leq \sup _{\left|\zeta^{\prime}\right| \leq \alpha}\left|h_{\sigma, \alpha}^{*}\left(\zeta-\zeta^{\prime}\right)\right| \cdot\left|\int_{\left|\zeta^{\prime}\right| \leq \alpha} \frac{\partial \psi_{\alpha}}{\partial \bar{\zeta}_{j}}\left(\zeta^{\prime}\right) d \zeta^{\prime} d \bar{\zeta}^{\prime}\right| \\
& \leq C_{1}\left(\left|h_{\sigma}^{*}(\zeta)\right|+2 m \alpha\right)
\end{aligned}
$$

where

$$
C_{1}:=\max \left(\max _{j}\left(\left|\int_{\left|\zeta^{\prime}\right| \leq \alpha} \frac{\partial \psi_{\alpha}}{\partial \bar{\zeta}_{j}}\left(\zeta^{\prime}\right) d \zeta^{\prime} d \bar{\zeta}^{\prime}\right|\right), 1\right) .
$$

For a $(p, q)$-form $g=\sum g_{I, J} d \zeta^{I} \wedge d \bar{\zeta}^{J}$, we denote its norm by $|g|:=\sup \left|g_{I, J}\right|$. Then we have

$$
\begin{aligned}
|w| \leq & |p| e^{\lambda(|\zeta|)-h_{\sigma, \alpha}^{*}(\zeta)} \\
\times & {\left[\left(\max _{|\tau| \leq 1+\varepsilon}\left|\frac{\partial \varphi}{\partial \tau}\right|+\max _{|\tau| \leq 1+\varepsilon}\left|\frac{\partial \varphi}{\partial \bar{\tau}}\right|\right)\left(\frac{1}{2}+C_{1}\left(2 m \alpha+\left|h_{\sigma}^{*}(\zeta)\right|\right)\right)\right.} \\
& \left.\times \max _{|\tau| \leq 1+\varepsilon}\left|\frac{\partial \varphi}{\partial \bar{\tau}}\right|\left|\frac{\partial \bar{\sigma}}{\sigma}\right|\right] \\
\leq & e^{H_{L}(\zeta)+\lambda(|\zeta|)-h_{\sigma, \alpha}^{*}(\zeta)} \max _{|\tau| \leq 1+\varepsilon}\left(\left|\frac{\partial \varphi}{\partial \tau}\right|,\left|\frac{\partial \varphi}{\partial \bar{\tau}}\right|\right) \\
& \times\left[1+2 C_{1}\left(2 m \alpha+\left|h_{\sigma}^{*}(\zeta)\right|\right)+2 e^{\lambda(|\zeta|)-h_{\sigma, \alpha}^{*}(\zeta)}|\partial \sigma|\right] .
\end{aligned}
$$

As $|\partial \sigma| \exp \left(-H_{-M}(\zeta)-\delta|\zeta|\right)$ is bounded, there exists a constant $C>0$ such that for $|\zeta| \gg 1$,

$$
\begin{aligned}
\log |w| & \leq H_{L}(\zeta)+H_{-M}(\zeta)+3 \delta|\zeta|-2 h_{\sigma}^{*}(\zeta)+\log |\zeta|+C \\
& \leq H_{L}(\zeta)-H_{-M}(\zeta)+4 \delta|\zeta|+C .
\end{aligned}
$$

In $Z_{1}$, from the estimate $H_{L}(\zeta)<H_{K}(\zeta)+\delta|\zeta|$, we get

$$
\log |w| \leq H_{K}(\zeta)-H_{-M}(\zeta)+5 \delta|\zeta|+C .
$$


In $Z_{3}$, there exists some constant $C_{2}>0$ such that

$$
\log |w| \leq C_{2} .
$$

Therefore we can take another constant $C^{\prime}>0$ such that the estimate

$$
\log |w| \leq H_{K}(\zeta)-H_{-M}(\zeta)+5 \delta|\zeta|+C^{\prime}
$$

holds in the whole $\mathbb{C}^{n}$. By the same argument as in Sébbar [Sé], we obtain the estimate

$$
\log |v| \leq H_{K}(\zeta)-H_{-M}(\zeta)+6 \delta|\zeta|+C_{3},
$$

for some constant $C_{3}>0$ (see [I-O1]).

We have

$$
\begin{aligned}
|q-v| & =\left|\frac{(1-\varphi) p}{\sigma}\right| \\
& = \begin{cases}0 & \text { if } \left.e^{\lambda(|\zeta|)-h_{\sigma, \alpha}^{*}(\zeta)}|\sigma(\zeta)| \leq 1 / 2\right), \\
2 e^{H_{L}(\zeta)+\lambda(|\zeta|)-h_{\sigma, \alpha}^{*}(\zeta)} & \text { (if } \left.e^{\lambda(|\zeta|)-h_{\sigma, \alpha}^{*}(\zeta)}|\sigma(\zeta)|>1 / 2\right),\end{cases}
\end{aligned}
$$

and so with a constant $C_{4}>0$, we have

$$
|q| \leq 2 \max (|q-v|,|v|) \leq \exp \left(H_{K \cup L}(\zeta)-H_{-M}(\zeta)+6 \delta|\zeta|+C_{4}\right) .
$$

For $r$, we remark that

$$
|p \varphi| \begin{cases}\leq e^{H_{L}(\zeta)} \leq e^{H_{K}(\zeta)+\delta|\zeta|} & \text { in } Z_{1}, \\ =0 & \text { in } Z_{2},\end{cases}
$$

and with a constant $C_{5}>0$,

$$
|\sigma v| \leq C_{5} e^{H_{K}(\zeta)+7 \delta|\zeta|}
$$

and thus we have

$$
|r| \leq \exp \left(H_{K}(\zeta)+7 \delta|\zeta|+C_{5}\right) .
$$

Thus taking $\delta>0$ so small that $7 \delta<\varepsilon$, we have the conclusion.

Now we can state our main theorem:

Theorem 4.2. Let $M \subset \mathbb{C}^{n}$ be a compact convex set and $S$ an analytic functional carried by $M$. Assume that $S$ satisfies the condition (S) in every direction in $\mathbb{C}^{n}$ and $h_{\hat{S}}^{*}(\zeta) \equiv H_{M}(\zeta)$. For an open convex set $\omega \subset \mathbb{C}^{n}$, let $\Omega$ be the open set defined by (4.1). Then any holomorphic solution $f(z)$ of the homogeneous convolution equation $S * f=0$ defined in $\omega+(-M)$ continues analytically to $\Omega+(-M)$.

Proof. We will prove that the restriction map

$$
\varrho: \mathcal{N}(\Omega+(-M)) \rightarrow \mathcal{N}(\omega+(-M))
$$


is an isomorphism. For the space $\mathcal{N}(\omega+(-M))$, we denote by $\mathcal{N}(\omega+(-M))^{\prime}$ the dual space. By Proposition 3.3, @ has dense image, so the transposed map ${ }^{t} \varrho: \mathcal{N}(\omega+(-M))^{\prime} \rightarrow \mathcal{N}(\Omega+(-M))^{\prime}$ is injective. It is sufficient to prove that ${ }^{t} \varrho$ is also surjective: By the Hahn-Banach theorem, every $T \in \mathcal{N}(\Omega+(-M))^{\prime}$ has an extension $T_{1} \in \mathcal{O}(\Omega+(-M))^{\prime}$. Then there exist a compact convex set $L \subset \Omega$ and a constant $C>0$ such that $\left|\hat{T}_{1}(\zeta)\right| \leq C e^{H_{L+(-M)}(\zeta)}$ for any $\zeta \in \mathbb{C}^{n}$. We can take a compact convex set $K \subset \omega$ satisfying (4.2). By applying Lemma 3.4 to $p:=\hat{T}_{1}, L+(-M)$ and $K+(-M)$, for any small $\varepsilon>0$, there exist entire functions $q(\zeta), r(\zeta)$ and a constant $C_{\varepsilon}>0$ such that

$$
\begin{aligned}
& p(\zeta)=\check{\hat{S}}(\zeta) q(\zeta)+r(\zeta), \\
& \log |q(\zeta)| \leq H_{L \cup K+(-M)}(\zeta)-H_{-M}(\zeta)+\varepsilon|\zeta|+C_{\varepsilon}=H_{L \cup K}(\zeta)+\varepsilon|\zeta|+C_{\varepsilon}, \\
& \log |r(\zeta)| \leq H_{K+(-M)}(\zeta)+\varepsilon|\zeta|+C_{\varepsilon} .
\end{aligned}
$$

Thus if $\varepsilon>0$ is taken small enough, we find analytic functionals $Q \in$ $\mathcal{O}(\Omega+(-M))^{\prime}$ and $R \in \mathcal{O}(\omega+(-M))^{\prime}$ corresponding to $q(\zeta)$ and $r(\zeta)$ (i.e. $\hat{Q}=q$ and $\hat{R}=r)$ such that $\hat{T}_{1}=\grave{\hat{S}} \hat{Q}+\hat{R}$. Then for any $g \in \mathcal{N}(\Omega+(-M))$, we have

$$
\langle T, g\rangle=\left\langle T_{1}, g\right\rangle=\langle Q, S * g\rangle+\langle R, g\rangle=\langle R, g\rangle,
$$

and this means $T={ }^{t} \varrho\left(R_{\mid \mathcal{N}(\omega+(-M))}\right)$.

5. Application to differential-difference operators in the complex domain. Let $\Lambda:=\left\{\lambda_{1}, \ldots, \lambda_{l}\right\}$ be a finite set in $\mathbb{C}^{n}, M$ its convex hull and $p_{j}(\zeta)$ an entire function of minimal type for $1 \leq j \leq l$. We denote by $S$ the analytic functional whose Fourier-Borel transform is $\sum_{j=1}^{l} p_{j}(\zeta) e^{\left\langle\zeta, \lambda_{j}\right\rangle}$. Then $S$ is carried by $M$. Furthermore by Theorem 6.1 .1 of Ronkin [R] and by [I-Oj], we know $h_{\hat{S}}^{*}(\zeta) \equiv H_{M}(\zeta)$ and that $\hat{S}(\zeta)$ satisfies the condition $(\mathrm{S})$ in every direction in $\mathbb{C}^{n}$. Thus $S$ satisfies all the hypotheses of Theorem 4.2.

In particular, in the case where $p_{j}$ 's are elliptic (i.e. their characteristic set is empty), we can prove that the characteristic set $\operatorname{Char}_{\infty}(S *)$ coincides with

$$
\left\{\zeta \infty \in S_{\infty}^{2 n-1}: \#\left\{j: \operatorname{Re}\left\langle\zeta, \lambda_{j}\right\rangle=H_{M}(\zeta)\right\} \geq 2\right\} .
$$

(See [I-O3] for more general results.) The figures below describe the case where $n=1$ and $j=1, M=$ the convex hull of $\Lambda$. We also remark that in this case, $\operatorname{Char}_{\infty}(S *)=$ the exterior normal directions $\left\{n_{1} \infty, n_{2} \infty, n_{3} \infty, n_{4} \infty\right\}$.

In Figure 2, every solution of $S * f=0$ holomorphic in $\omega+(-M)$ continues analytically to each of the four corners. 

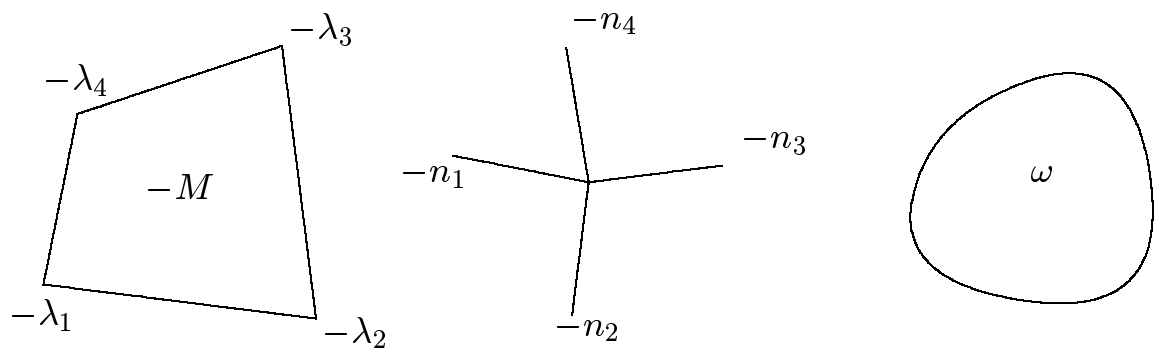

Fig. 1. $-M, \operatorname{Char}(S *)^{a}$ and $\omega$

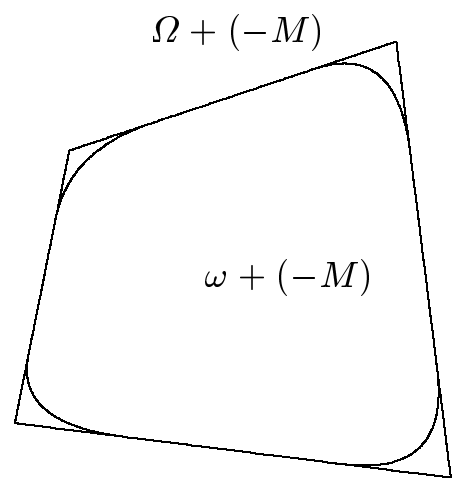

Fig. 2. $\omega+(-M)$ and $\Omega+(-M)$

\section{References}

[A] T. A oki, Existence and continuation of holomorphic solutions of differential equations of infinite order, Adv. in Math. 72 (1988), 261-283.

[I1] R. Ishimura, A remark on the characteristic set for convolution equations, Mem. Fac. Sci. Kyushu Univ. 46 (1992), 195-199.

[I2] - , The characteristic set for differential-difference equations in real domains, Kyushu J. Math. 53 (1999), 107-114.

[I-O1] R. Ishimura and Y. Okada, The existence and the continuation of holomorphic solutions for convolution equations in tube domains, Bull. Soc. Math. France 122 (1994), 413-433.

[I-O2] - - - The micro-support of the complex defined by a convolution operator in tube domains, in: Singularities and Differential Equations, Banach Center Publ. 33, Inst. Math., Polish Acad. Sci., 1996, 105-114.

[I-O3] -, -, Examples of convolution operators with described characteristics, in preparation.

[I-Oj] - - - Sur la condition (S) de Kawai et la propriété de croissance régulière d'une fonction sous-harmonique et d'une fonction entière, Kyushu J. Math. 48 (1994), $257-263$. 
[Ka] T. Kawai, On the theory of Fourier hyperfunctions and its applications to partial differential equations with constant coefficients, J. Fac. Sci. Univ. Tokyo Sect. IA Math. 17 (1970), 467-517.

[Ki] C. O. Kiselman, Prolongement des solutions d'une équation aux dérivées partielles à coefficients constants, Bull. Soc. Math. France 97 (1969), 329-356.

[Ko] Yu. F. Korobeřnik, Convolution equations in the complex domain, Math. USSR-Sb. 36 (1985), 171-193.

[Kr] A. S. Krivosheev, A criterion for the solvability of nonhomogeneous convolution equations in convex domains of $\mathbb{C}^{n}$, Math. USSR-Izv. 36 (1991), 497-517.

[Ll-G] P. Lelong and L. Gruman, Entire Functions of Several Complex Variables, Grundlehren Math. Wiss. 282, Springer, Berlin, 1986.

[Lv] B. Ja. Levin, Distribution of Zeros of Entire Functions, Transl. Math. Monographs, Amer. Math. Soc., Providence, 1964.

[R] L. I. Ronkin, Functions of Completely Regular Growth, Kluwer, 1992.

[Sé] A. Sébbar, Prolongement des solutions holomorphes de certains opérateurs différentiels d'ordre infini à coefficients constants, in: Séminaire Lelong-Skoda, Lecture Notes in Math. 822, Springer, Berlin, 1980, 199-220.

[V] A. Vidras, Interpolation and division problems in spaces of entire functions with growth conditions and their applications, Doct. Diss., Univ. of Maryland.

[Z] M. Zerner, Domaines d'holomorphie des fonctions vérifiant une équation aux dérivées partielles, C. R. Acad. Sci. Paris 272 (1971), 1646-1648.

Ryuichi Ishimura and Yasunori Okada

Jun-ichi Okada

Department of Mathematics and Informatics

Faculty of Sciences

Institute of Natural Sciences

Chiba University

Yayoi-cho, Inage-ku, Chiba 263-8522, Japan

Yayoi-cho, Inage-ku, Chiba 263-8522, Japan

E-mail: ishimura@math.s.chiba-u.ac.jp

okada@math.s.chiba-u.ac.jp

Reçu par la Rédaction le 8.12.1998

Révisé le 28.7.1999 et 5.9.2000 